\title{
Preparation of microstructured hydroxyapatite microspheres using oil in water emulsions
}

\author{
T S PRADEESH, M C SUNNY, H K VARMA and P RAMESH* \\ Biomedical Technology Wing, Sree Chitra Tirunal Institute for Medical Sciences and Technology, \\ Thiruvananthapuram 695 012, India
}

MS received 31 July 2004; revised 30 April 2005

\begin{abstract}
Hydroxyapatite (HAP) microspheres with peculiar spheres-in-sphere morphology were prepared by using oil-in-water emulsions and solvent evaporation technique. Ethylene vinyl acetate co-polymer (EVA) was used as the binder material. Preparation of HAP/EVA microspheres was followed by the thermal debinding and sintering at $1150^{\circ} \mathrm{C}$ for $3 \mathrm{~h}$ to obtain HAP microspheres. Each microsphere of 100-1000 $\mu \mathrm{m}$ was in turn composed of spherical hydroxyapatite granules of $2-15 \mu \mathrm{m}$ size which were obtained by spray drying the precipitated HAP. The parameters such as percentage of initial HAP loading, type of stabilizer, concentration of stabilizer, stirring speed and temperature of microsphere preparation were varied to study their effect on the particle size and geometry of the microspheres obtained. It was observed that these parameters do have an effect on the size and shape of the microspheres obtained, which in turn will affect the sintered HAP microstructure. Of the three stabilizers used viz. polyoxyethylene(20) sorbitan monopalmitate (Tween-40), sodium laurate and polyvinyl alcohol (PVA), only PVA with a concentration not less than $0 \cdot 1 \mathrm{wt} \%$ showed controlled stabilization of HAP granules resulting in spherical microspheres of required size. Morphologically better spherical microspheres were obtained at $20^{\circ} \mathrm{C}$. Increasing the stirring speed produced smaller microspheres. Smaller microspheres having size $<50 \mu \mathrm{m}$ were obtained at a stirring speed of $1500 \pm 50 \mathrm{rpm}$. A gradual decrease in pore size was observed in the sintered microspheres with increase in HAP loading.
\end{abstract}

Keywords. Ethylene vinyl acetate co-polymer; hydroxyapatite; microstructured microspheres; sintering.

\section{Introduction}

Synthetic hydroxyapatite (HAP), having the chemical formula, $\mathrm{Ca}_{10}\left(\mathrm{PO}_{4}\right)_{6}(\mathrm{OH})_{2}$, is emerging as the most promising bioceramic material for orthopaedic applications because of its similarity in chemical structure to that of the inorganic matrix of the living bone and teeth (Jarcho et al 1976; Shigeru et al 1989). Hydroxyapatite, having excellent bonding ability to the host bone does not show any foreign body reactions (Bonfield et al 1981; Klein et al 1989).

The present biomedical scenario sees the increased use of HAP, in different forms, for various applications. Of these, particulate forms have received much attention due to their specific applications. Matsumoto et al (2004) suggested that HAP particles synthesized at different temperatures are useful as controlled release carriers of protein. Kunieda et al (1993) used HAP spheres, with diameter $1000 \mu \mathrm{m}$, for sustained release of doxorubicin by direct implantation into hepatic tumours in mice and rabbits. Hydroxyapatite particles have also been utilized for purification of nucleic acid (Kumazawa et al 1992).

\footnotetext{
*Author for correspondence (rameshp@sctimst.ker.nic.in; rameshsct@yahoo.com)
}

Various studies have been conducted using different polymers such as gelatin, collagen, chitosan etc as matrices for the preparation of HAP microspheres (Hsu et al 1999; Paul and Sharma 1999; Sivakumar and Rao 2002; Sunny et al 2002; Wang et al 2003; Wu et al 2004). Velayudhan et al (2000) tried ethylene vinyl acetate copolymer (EVA) as a binder material to prepare intricate shapes of HAP for various bone substitute applications.

In the present study we report the preparation and characterization of porous HAP microspheres using EVA as the binder. The aim of this work is to prepare hydroxyapatite microspheres of definite size with sphere in sphere morphology by optimizing the stirring speed, stabilizer concentration, sintering temperature and sintering time for specific applications such as bone filling, drug delivery etc.

\section{Materials and methods}

Ethylene vinyl acetate (EVA) copolymer having weight average molecular weight, 84066, $28 \mathrm{wt} \%$ vinyl acetate content and melt flow index value of 25 was procured from M/s NOCIL, Chennai. Tween-40, sodium laurate and cold water soluble PVA (weight average molecular weight, 14000) was obtained from SD fine chemicals, 
Mumbai. The other chemicals used in the present study were of analytical grade and used as received.

\subsection{Preparation and characterization of spherical HAP granules}

Spherical HAP granules of $2-15 \mu \mathrm{m}$ were synthesized by the precipitation reaction between ammoniated calcium nitrate and dihydrogen phosphate solution according to the procedure described elsewhere (Varma and Sivakumar 1996). The precipitated HAP was aged in the mother liquor for $24 \mathrm{~h}$. Then it was washed and filtered to remove the soluble by-products. The filter cake was then dispersed in distilled water to get a slurry containing $15 \mathrm{wt} \%$ HAP and spray dried using BUCHI B191 Spray Dryer, Switzerland, with a maximum throughput of dry air $35 \mathrm{~m}^{3} / \mathrm{h}$, and at a chamber temperature of $90-92^{\circ} \mathrm{C}$. The morphology of the HAP granules prepared was examined using a scanning electron microscope (Hitachi Model S-2400, Japan) and the particle size analysis of the granules was carried out using a Malvern Mastersizer 2000 particle size analyser (Malvern Instruments, UK).

\subsection{Preparation of microspheres}

Initially, a solution of $5 \mathrm{wt} \%$ EVA in dichloromethane (DCM) was prepared. The requisite amount (50-80 wt\%), of spray dried HAP granules was mixed with $10 \mathrm{ml} \mathrm{EVA}$ solution by using a magnetic stirrer in a $25 \mathrm{ml}$ stoppered conical flask. The mixture was then dispersed into $200 \mathrm{ml}$ of distilled water containing different concentrations of PVA ranging from $0-5 \mathrm{wt} \%$ as the stabilizer. The system was kept stirred at different revolutions (ranging from 200-1500 rpm) with a motor driven half moon paddle stainless steel stirrer (diameter, $5 \mathrm{~cm}$ ) at different temperatures $\left(20,30\right.$ and $\left.40^{\circ} \mathrm{C}\right)$. The stirring was continued for 3-4 h to facilitate the evaporation of DCM. The microspheres obtained were filtered, washed several times with distilled water and finally the microspheres were dried in an air oven at $60^{\circ} \mathrm{C}$ to constant weight.

The effect of different stabilizers (Tween-40, sodium laurate and polyvinyl alcohol), stabilizer concentration, stirring speed and temperature on the particle size and particle geometry of HAP/EVA microspheres was studied.

\subsection{Thermogravimetric analysis}

The thermogravimetric analysis was conducted using SDT-2960 simultaneous DTA-TGA analyser to study the thermal degradation behaviour of EVA in the microspheres and to determine the actual incorporation of HAP in the microspheres. Here the weight loss of the microspheres with 50 and 70 weight percentage filler (HAP) were measured continuously by heating the sample from $25-800^{\circ} \mathrm{C}$ at a rate of $10^{\circ} \mathrm{C} / \mathrm{min}$.

\subsection{Thermal debinding and sintering process of HAP/EVA microspheres}

The thermal debinding and sintering of HAP/EVA microspheres were carried out in a high temperature furnace (OKAY Raising Hearth High Temperature Furnace, Kolkata, India). The heating of the green microspheres was programmed in such a manner that the rate of heating was kept at $2^{\circ} \mathrm{C} / \mathrm{min}$ up to a temperature of $700^{\circ} \mathrm{C}$ and held at this temperature for $2 \mathrm{~h}$. The microspheres were then heated rapidly to $1150^{\circ} \mathrm{C}$ and sintering was continued at this temperature for $3 \mathrm{~h}$. Then they were cooled to room temperature by keeping the sample in the furnace itself.

\subsection{Morphological studies}

Morphological investigation of HAP/EVA microspheres with different HAP contents prepared at $20^{\circ} \mathrm{C}$ was carried out before and after sintering, using scanning electron microscope (Hitachi Model S-2400, Japan). The microspheres were sputter-coated with gold before examination under the scanning electron microscope (SEM).

\section{Results and discussion}

\subsection{Preparation of HAP granules}

The size of the spherical HAP granules obtained by the precipitation method followed by spray drying was in the range between 2 and $15 \mu \mathrm{m}$ as shown in figure 1a. The particle size distribution of the spray dried HAP granules showed $d_{0.1}, d_{0.5}$ and $d_{0.9}$ values to be $2.21 \mu \mathrm{m}, 5.84 \mu \mathrm{m}$ and $14.02 \mu \mathrm{m}$, respectively. $d_{0.1}, d_{0.5}$ and $d_{0.9}$ denote the diameter of the granules at share $\% 10,50$ and 90, respectively. It has further been observed from the SEM picture of spray dried HAP granules that each spherical granule is a cluster of many basic crystallites of HAP having the grain size between 80 and $100 \mathrm{~nm}$ (figure 1b).

\subsection{Preparation of microspheres}

Microspheres were prepared by oil-in-water emulsion followed by solvent evaporation technique. Oil-in-water emulsion was obtained by dispersing the organic phase (dichloromethane solution containing $5 \mathrm{wt} \%$ of EVA and appropriate amount of HAP) in the aqueous medium of the surfactant. While dispersing in aqueous phase, the organic phase was transformed into tiny droplets and each droplet was surrounded by surfactant molecules. The protective layer thus formed on the surface prevented the droplets from coalescing and helped to stay individual droplets. While stirring, DCM was slowly evaporated from the droplets and after the complete removal of 
DCM, the droplets solidified to become individual microspheres. The size of the droplets formed depends on many factors like type and concentration of the stabilizing agents, on the type and speed of stirring employed etc, which in turn affects the size of the final microspheres formed.

\subsection{Effect of different stabilizers}

The effect of three stabilizers viz. Tween-40, sodium laurate and PVA, on the size and geometry of the microspheres was studied by keeping the other parameters like
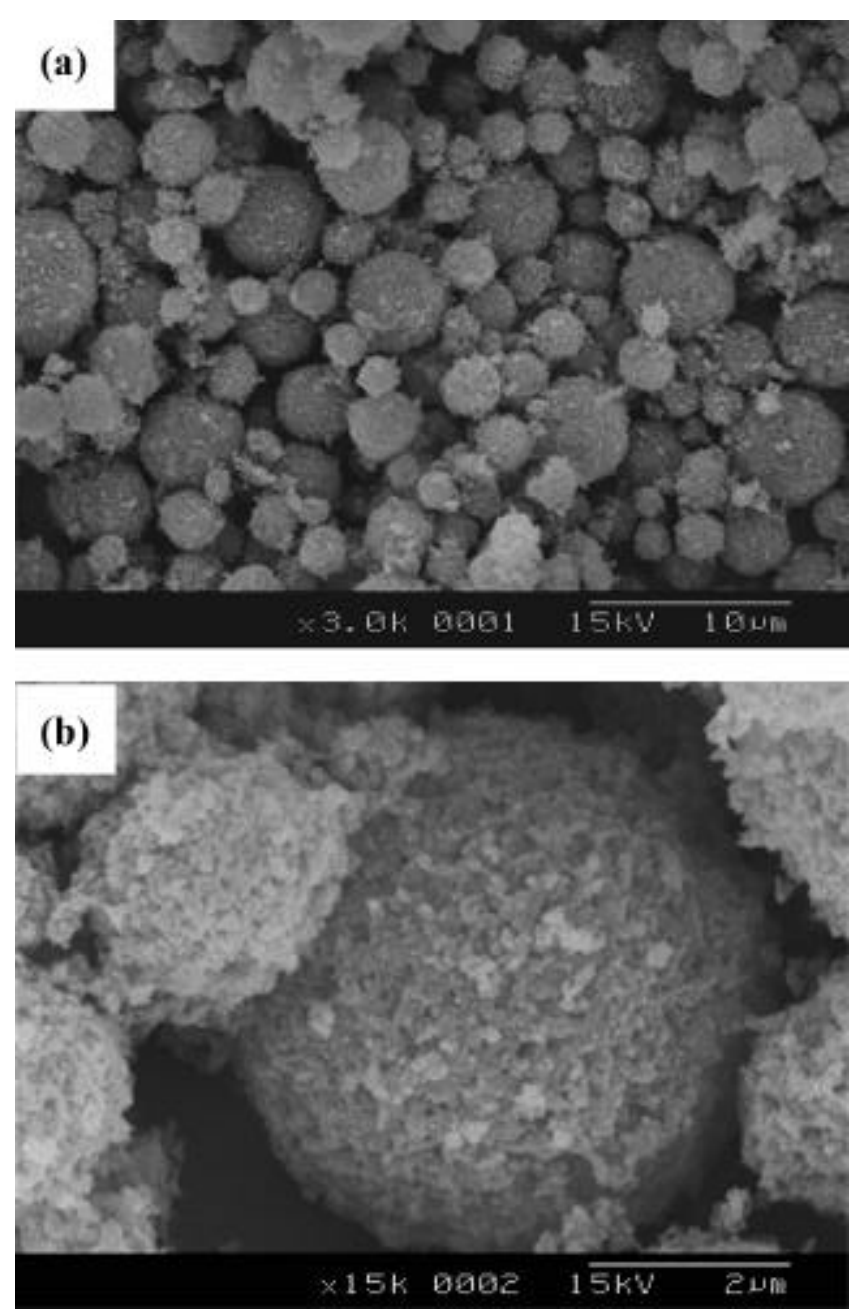

Figure 1. SEM micrographs of spray dried HAP powder at (a) $\times 3 \mathrm{k}$ and $(\mathbf{b}) \times 15 \mathrm{k}$. the stirring speed $(500 \pm 50 \mathrm{rpm})$, stabilizer concentration $(0.1 \mathrm{wt} \%)$, HAP content $(50 \mathrm{wt} \%)$ and temperature $\left(20^{\circ} \mathrm{C}\right)$ constant. The results summarized in table 1 show that only PVA gave spherical spheres without agglomeration. Further it has been seen that in the case of Tween-40 and sodium laurate, the increase of stabilizer concentration up to $1 \mathrm{wt} \%$ has no effect on the microsphere formation.

\subsection{Effect of stabilizer concentrations}

The effect of different concentrations of PVA, from 0$5 \mathrm{wt} \%$ as mentioned in table 2, on the size and nature of the microspheres was studied by keeping the stirring speed at $500 \pm 50 \mathrm{rpm}$ and temperature, $20^{\circ} \mathrm{C}$ at a $\mathrm{HAP}$ content of $50 \mathrm{wt} \%$. The results indicate that the variation in the concentration of PVA in the dispersion medium affects the size of the microspheres. It was observed that the minimum concentration of PVA required for the stabilization of organic droplets in the aqueous medium was $0 \cdot 1 \mathrm{wt} \%$. Below this value, the droplets were not stabilized and finally adhered on the stirrer. The results in table 2 indicate that as the percentage of PVA increases, the size of the microspheres decreases. At $0 \cdot 1 \mathrm{wt} \%$ PVA, most of the microspheres were in the range $200-500 \mu \mathrm{m}$. However, at $0.5 \mathrm{wt} \% \mathrm{PVA}$, microspheres in the range 100-200 $\mu \mathrm{m}$ were obtained. Microspheres with size $<100 \mu \mathrm{m}$ could be obtained with $1-2 \mathrm{wt} \%$ stabilizer. It should be mentioned that at $5 \mathrm{wt} \%$ of PVA, there was a drastic reduction in the size of the microspheres $(\sim 20 \mu \mathrm{m})$.

\subsection{Effect of stirring speed}

The effect of various stirring speeds on the particle size of the microspheres was studied using $0 \cdot 1 \mathrm{wt} \%$ PVA stabilizer at a temperature of $20^{\circ} \mathrm{C}$ and $50 \mathrm{wt} \%$ HAP content. The results summarized in table 3 show that the increase in the stirring speed causes a decrease in the size of the microspheres. This is because of the breaking down of the larger droplets of the dispersed phase into smaller droplets with increase in stirring speed.

\subsection{Effect of temperature}

Experiments were conducted at three different temperatures, $20^{\circ} \mathrm{C}, 30^{\circ} \mathrm{C}$ and $40^{\circ} \mathrm{C}$, keeping the PVA concentration at $0.1 \mathrm{wt} \%$, stirring speed of $500 \pm 50 \mathrm{rpm}$ and $50 \mathrm{wt} \%$

Table 1. Effect of different stabilizers on the particle geometry.

\begin{tabular}{lccccc}
\hline Stabilizer & $\begin{array}{c}\text { Quantity of the } \\
\text { stabilizer }(\mathrm{wt} \%)\end{array}$ & $\begin{array}{c}\text { Stirring speed } \\
(\mathrm{rpm})\end{array}$ & $\begin{array}{c}\text { Temperature } \\
\left({ }^{\circ} \mathrm{C}\right)\end{array}$ & $\begin{array}{c}\text { HAP content } \\
(\%)\end{array}$ & $\begin{array}{c}\text { Shape of the } \\
\text { microspheres }\end{array}$ \\
\hline Tween-40 & $0 \cdot 10$ & $500 \pm 50$ & 20 & 50 & Irregular \\
Sodium laurate & $0 \cdot 10$ & $500 \pm 50$ & 20 & 50 & Irregular \\
PVA & $0 \cdot 10$ & $500 \pm 50$ & 20 & 50 & Spherical \\
\hline
\end{tabular}


HAP content. Only the microspheres produced at $20^{\circ} \mathrm{C}$ showed perfect spherical shape compared to that produced at 30 or $40^{\circ} \mathrm{C}$. This is mainly due to the slow evaporation of DCM from the droplets and hence better stabilization which in turn retains the shape of the microspheres intact. At elevated temperature like 30 and $40^{\circ} \mathrm{C}$, the geometry of the microspheres was affected due to the higher evaporation rate of DCM. Due to the higher evaporation rate, the dispersed phase does not get enough time to become spherical droplets before solidification.

\subsection{Morphological evaluation of HAP/EVA micro- spheres}

The SEM micrographs of HAP/EVA microspheres containing $70 \mathrm{wt} \%$ of HAP at different magnifications are shown in figures $2(a)-(c)$. The higher magnification pictures show that each microsphere is composed of tiny HAP granules of size $2-15 \mu \mathrm{m}$ embedded in the EVA matrix. The uniform distribution of HAP granules in the EVA matrix is clearly evident from the micrographs of figures 2(b)-(c).

\subsection{Thermogravimetric analysis}

Thermograms of 50 and $70 \mathrm{wt} \%$ HAP/EVA microspheres are shown in figure 3. The thermograms show a two-step binder burn out schedule. As indicated by many research groups, EVA upon heating undergoes a side group elimination reaction which generates acetic acid and a copolymer, polyethylene co-polyacetylene having double bond in the chain (Jellinek 1955; Hrdina et al 1998; Trunec and Cihlar 2002). The weight loss in the first stage of EVA copolymer degradation (at around $250-350^{\circ} \mathrm{C}$ ) was due to the elimination of side acetate groups in vinyl acetate unit. The second stage degradation pattern in the thermogram at elevated temperature $\left(400-500^{\circ} \mathrm{C}\right)$ was due to the degradation of polyethylene co-polyacetylene copolymer by chain scission. It can further be seen that the degradation behaviour does not change as the filler content is increased. But the percentage loss mainly depends upon the amount of binder.

The actual incorporation efficiency of HAP in the microspheres could also be determined from the thermogravimetric analysis. The thermograms of 50 and $70 \mathrm{wt} \% \mathrm{HAP} /$ EVA microspheres indicate that only 44.5 and $61.6 \mathrm{wt} \%$ of the total weight of the microspheres, respectively remain after the removal of the polymer. The incorporation efficiency of HAP was thus found to be $89 \%$ and $88 \%$ in the case of 50 and $70 \mathrm{wt} \%$ HAP incorporated microspheres, respectively.

\subsection{Thermal de-binding and sintering}

Thermal de-binding removes the polymer from the microspheres gradually. At around $500^{\circ} \mathrm{C}$ the polymeric binder will be degraded and burned out from the microspheres. By increasing the temperature rapidly to $1150^{\circ} \mathrm{C}$, sintering of individual HAP granules present within the spheres will be taking place, while retaining the shape of the microspheres intact. This can be evidenced from the SEM photograph. Figures 4(a)-(d) show the SEM micrographs

Table 2. Effect of PVA concentration on particle size of microspheres.

\begin{tabular}{lcccc}
\hline $\begin{array}{l}\text { PVA concentration } \\
(\mathrm{wt} \%)\end{array}$ & $\begin{array}{c}\text { Stirring speed } \\
(\mathrm{rpm})\end{array}$ & $\begin{array}{c}\text { Temperature } \\
\left({ }^{\circ} \mathrm{C}\right)\end{array}$ & $\begin{array}{c}\text { HAP content } \\
(\mathrm{wt} \%)\end{array}$ & $\begin{array}{c}\text { Microsphere size } \\
\text { range }(\mu \mathrm{m})\end{array}$ \\
\hline 0 & $500 \pm 50$ & 20 & 50 & - \\
$0 \cdot 05$ & $500 \pm 50$ & 20 & 50 & - \\
$0 \cdot 1$ & $500 \pm 50$ & 20 & 50 & $100-500$ \\
$0 \cdot 5$ & $500 \pm 50$ & 20 & 50 & $<200$ \\
$1 \cdot 0$ & $500 \pm 50$ & 20 & 50 & $<100$ \\
$2 \cdot 0$ & $500 \pm 50$ & 20 & 50 & $60-40$ \\
$3 \cdot 0$ & $500 \pm 50$ & 20 & 50 & $50-20$ \\
$4 \cdot 0$ & $500 \pm 50$ & 20 & 50 & $\sim 20$ \\
$5 \cdot 0$ & $500 \pm 50$ & 20 & 50 & \\
\hline
\end{tabular}

Table 3. Effect of stirring speed on the particle size of microspheres.

\begin{tabular}{lcccc}
\hline $\begin{array}{l}\text { Stirring speed } \\
(\mathrm{rpm})\end{array}$ & $\begin{array}{c}\text { PVA concentration } \\
(\mathrm{wt} \%)\end{array}$ & $\begin{array}{c}\text { Temperature } \\
\left({ }^{\circ} \mathrm{C}\right)\end{array}$ & $\begin{array}{c}\text { HAP content } \\
(\mathrm{wt} \%)\end{array}$ & $\begin{array}{c}\text { Microsphere size } \\
\text { range }(\mu \mathrm{m})\end{array}$ \\
\hline $1500 \pm 50$ & $0 \cdot 1$ & 20 & 50 & $<50$ \\
$750 \pm 50$ & $0 \cdot 1$ & 20 & 50 & $<100$ \\
$500 \pm 50$ & $0 \cdot 1$ & 20 & 50 & $100-500$ \\
$200 \pm 50$ & $0 \cdot 1$ & 20 & 50 & $200-1000$ \\
\hline
\end{tabular}


of HAP microspheres obtained after sintering HAP/EVA microspheres with $50-80 \mathrm{wt} \%$ of HAP. These micrographs indicate that microspheres with 50\% HAP are perfectly spherical in shape with size ranging between
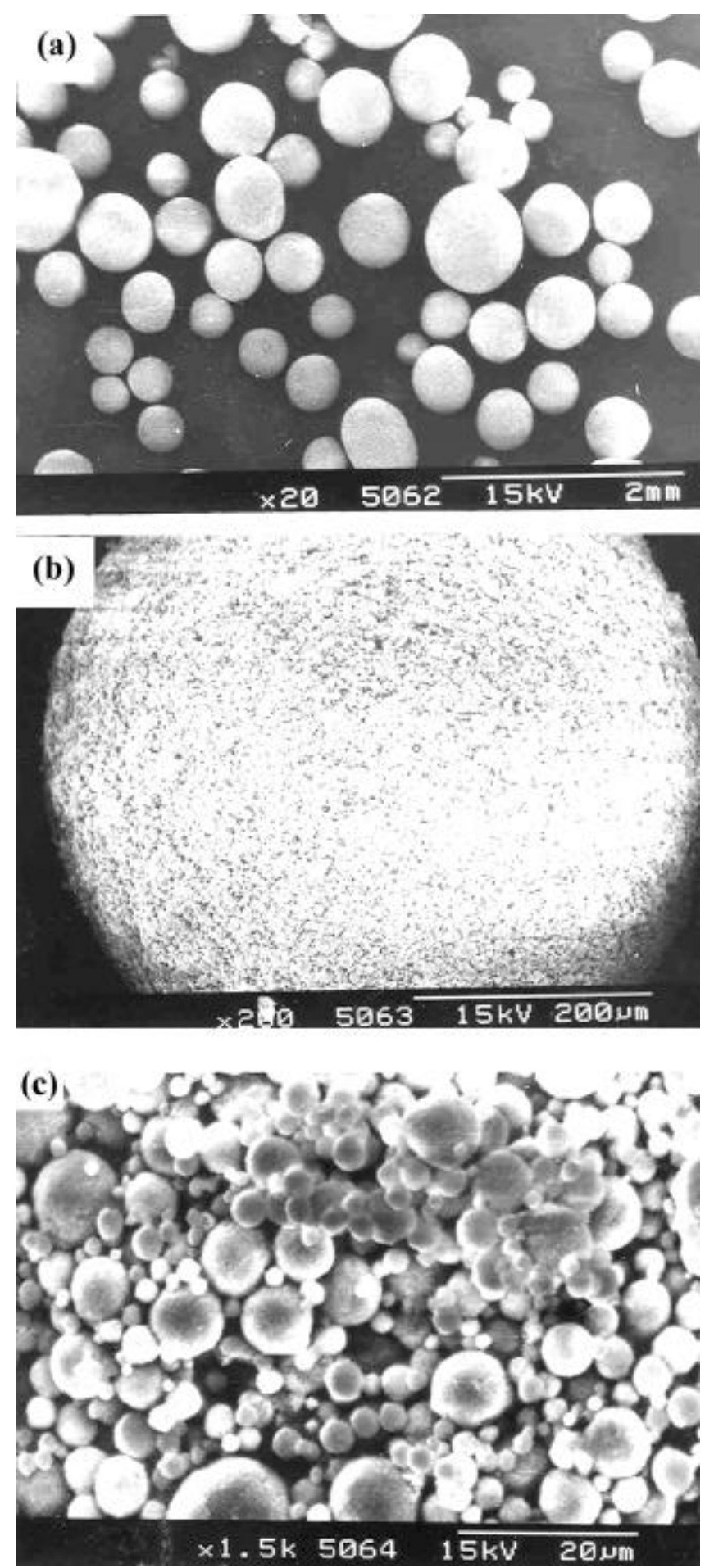

Figure 2. SEM micrographs of green HAP/EVA microspheres prepared at $500 \pm 50 \mathrm{rpm}, 20^{\circ} \mathrm{C}$ and $0.1 \mathrm{wt} \%$ PVA concentration containing $70 \mathrm{wt} \%$ of HAP at different magnifications: (a) $\times 20,($ b) $\times 200$ and $(\mathbf{c}) \times 1.5 \mathrm{k}$.
200 and $500 \mu \mathrm{m}$ and as the wt $\%$ of HAP was increased to 60 and $70 \%$, most of the spheres retained the spherical shape but the size of the microspheres increased to 500$1000 \mu \mathrm{m}$. This increase in size with increase in HAP content is mainly due to the higher viscosity of the dispersed phase. Due to the higher viscosity, the breakdown of the larger droplets into smaller ones is difficult at a slower stirring speed of $500 \pm 50 \mathrm{rpm}$. It should be mentioned that there was no control over the size and shape of the microspheres beyond $70 \mathrm{wt} \%$ HAP loading. And it was mainly due to the very high viscosity of the dispersed phase as well as the low concentration of the binder (EVA) to hold the HAP granules intact as a droplet in the dispersing medium.

\subsection{Porosity}

The SEM micrographs in figures 5(a)-(d) show the high magnification surface characteristic of HAP microspheres obtained by sintering HAP/EVA microspheres with different HAP contents. It can be seen that each sphere is about $200-800 \mu \mathrm{m}$ in size consisting of smaller spherical HAP granules of $2-15 \mu \mathrm{m}$. Therefore, these HAP microspheres having spheres-in-sphere morphology can be designated as 'microstructured microspheres'. This microstructured spheres-in-sphere morphology is attained at a low sintering temperature of $1150^{\circ} \mathrm{C}$ for about $3 \mathrm{~h}$.

From figures $5(\mathrm{a})-(\mathrm{d})$, it can be seen that the porosity of microspheres decreases when the percentage of HAP in HAP/EVA microspheres increases from $50-80 \mathrm{wt} \%$. The decrease in porosity of the higher HAP loaded microspheres is mainly due to the increase in the HAP/ polymer ratio in the dispersed phase. As the HAP loading increases, the packing of HAP granules increases in a microsphere which in turn improves the sintering and pore size/volume reduction.

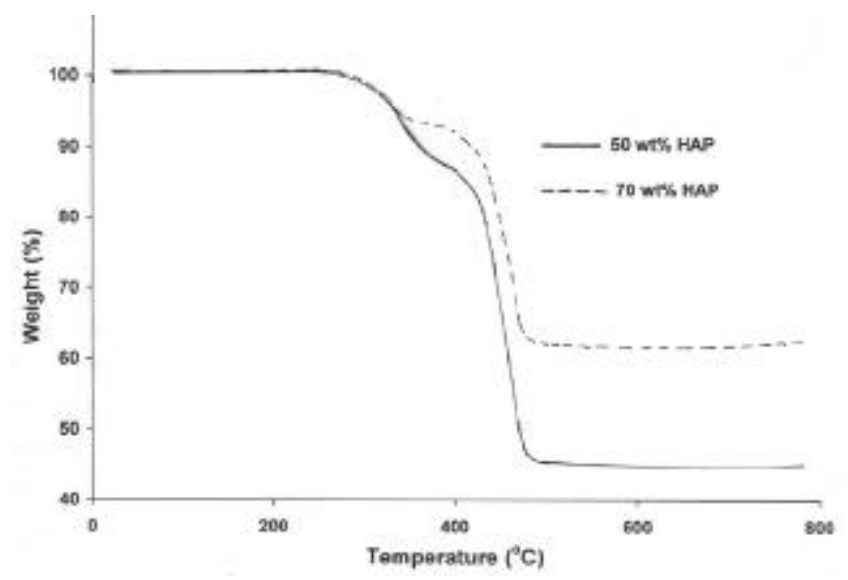

Figure 3. Thermograms of 50 and $70 \mathrm{wt} \%$ HAP/EVA microspheres prepared at $500 \pm 50 \mathrm{rpm}, 20^{\circ} \mathrm{C}$ and $0.1 \mathrm{wt} \%$ PVA concentration. 

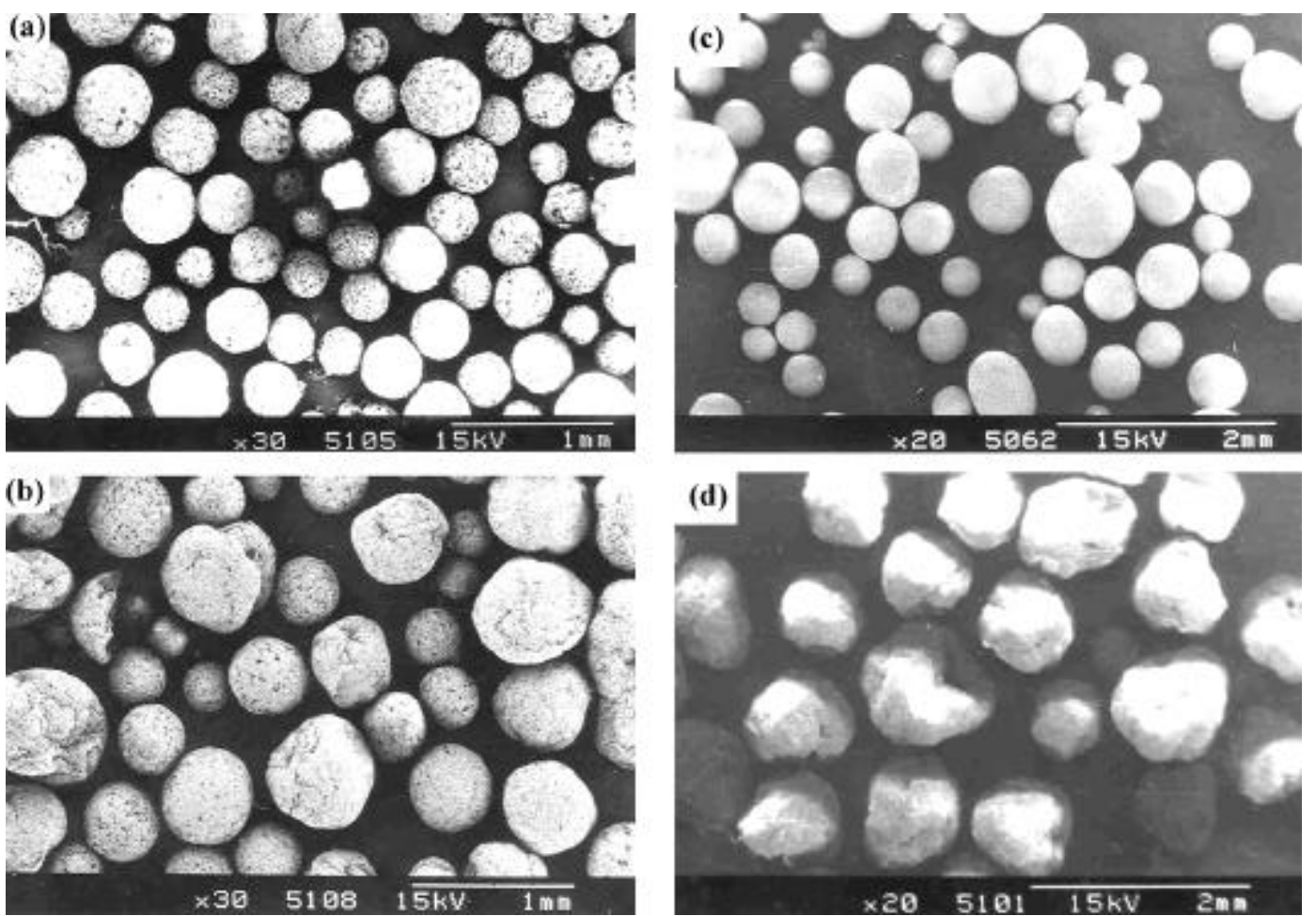

Figure 4. SEM micrographs of sintered HAP/EVA microspheres prepared at $500 \pm 50 \mathrm{rpm}, 20^{\circ} \mathrm{C}$ and $0.1 \mathrm{wt} \%$ PVA concentration containing (a) $50 \mathrm{wt} \%$ of HAP, (b) $60 \mathrm{wt} \%$ of HAP, (c) $70 \mathrm{wt} \%$ of HAP and (d) $80 \mathrm{wt} \%$ of HAP.
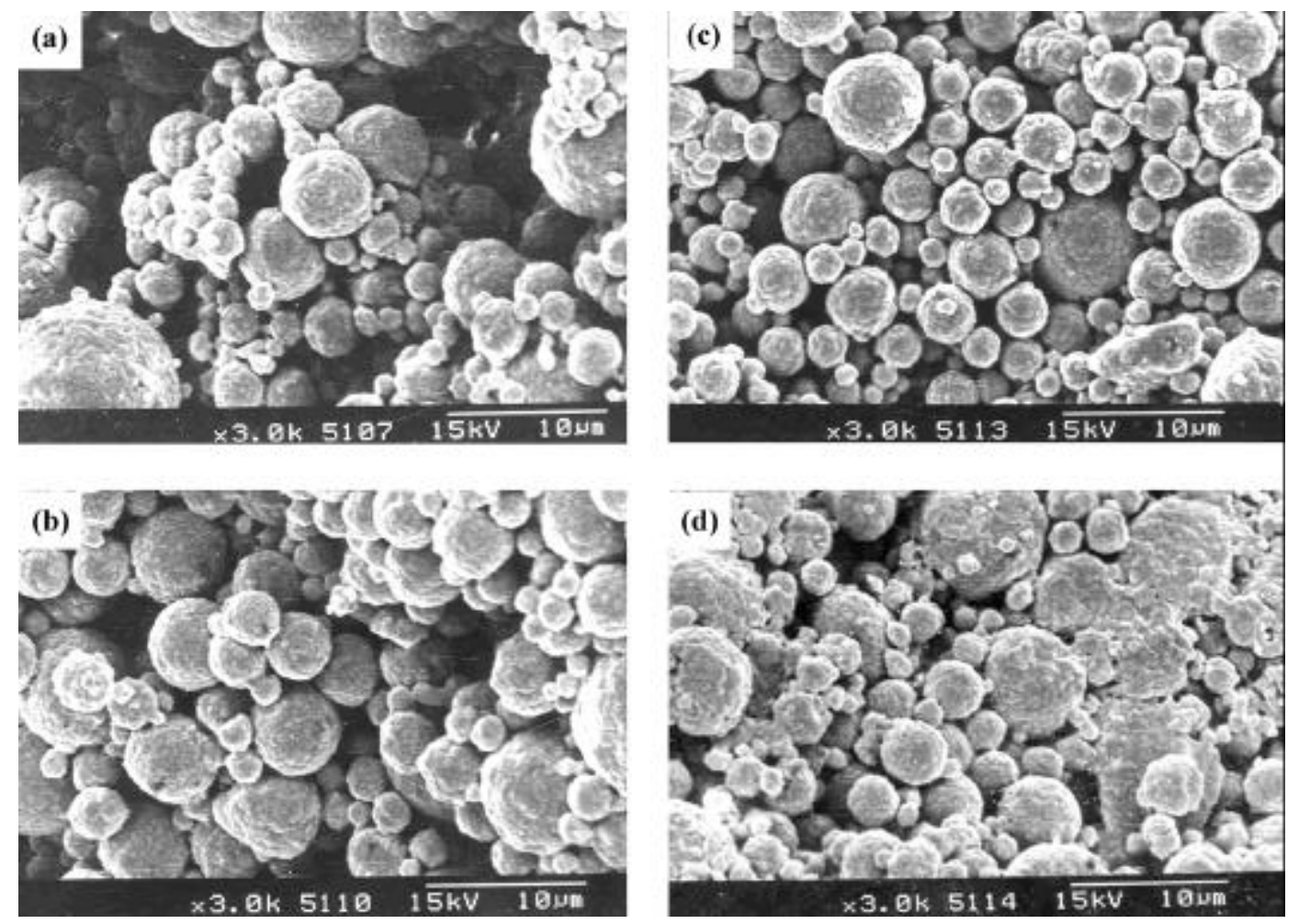

Figure 5. SEM micrographs of sintered HAP/EVA microspheres prepared at $500 \pm 50 \mathrm{rpm}, 20^{\circ} \mathrm{C}$ and $0.1 \mathrm{wt} \%$ PVA concentration containing (a) $50 \mathrm{wt} \%$ of HAP $(\times 3.0 \mathrm{k})$, (b) $60 \mathrm{wt} \%$ of HAP $(\times 3.0 \mathrm{k})$, (c) $70 \mathrm{wt} \%$ of HAP $(\times 3.0 \mathrm{k})$ and $(\mathbf{d}) 80 \mathrm{wt} \%$ of HAP $(\times 3.0 \mathrm{k})$. 

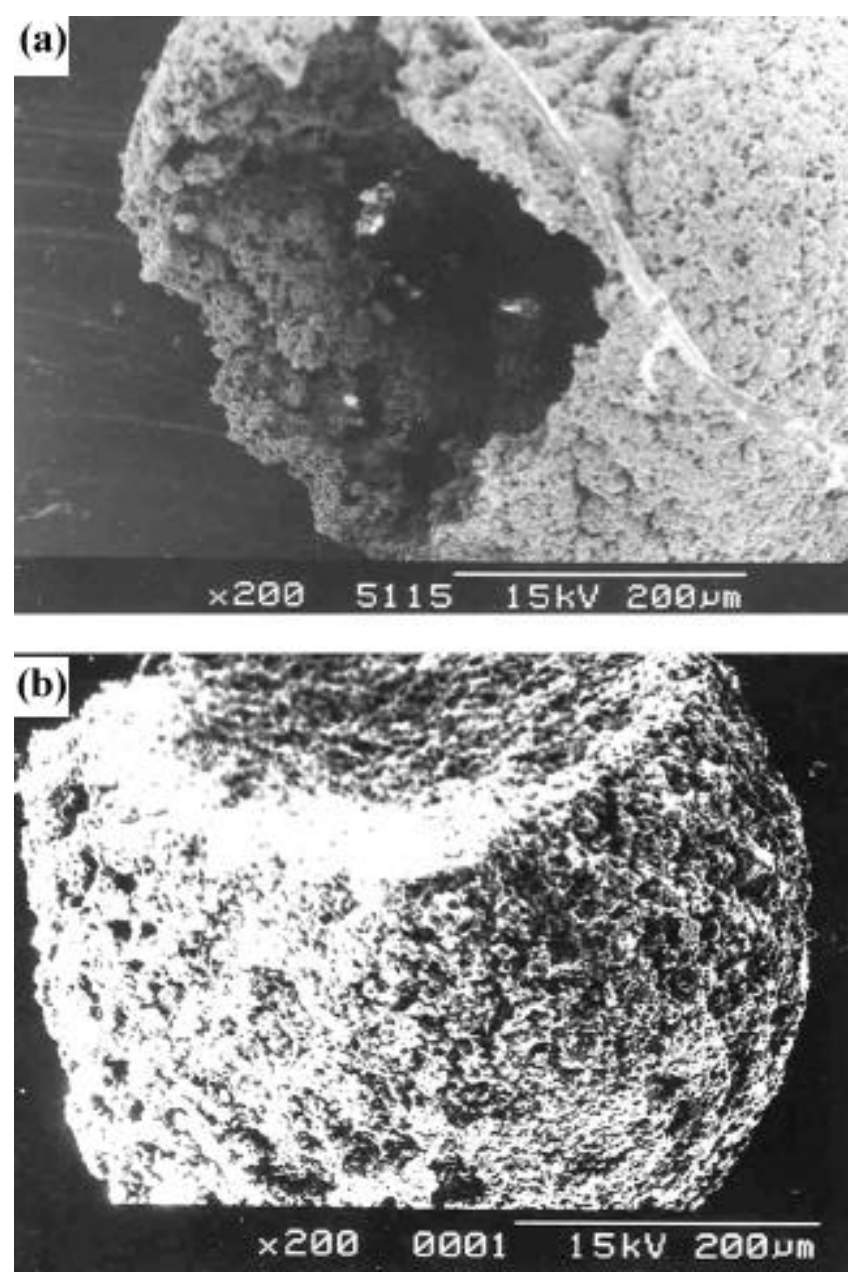

Figure 6. SEM micrographs showing the fracture morphology of (a) $50 \mathrm{wt} \%$ and (b) $70 \mathrm{wt} \%$ HAP loaded microspheres prepared at $500 \pm 50 \mathrm{rpm}, 20^{\circ} \mathrm{C}$ and $0 \cdot 1 \mathrm{wt} \%$ PVA concentration.

\subsection{Fracture morphology}

Figures 6(a) and (b) show the fracture morphology of 50 and $70 \mathrm{wt} \%$ HAP loaded microspheres, respectively. Fracture morphology studies reveal that $50 \mathrm{wt} \%$ loaded HAP contains both dense and hollow spheres, whereas only dense spheres were obtained with $70 \mathrm{wt} \%$ HAP loading. It has been observed that in the case of microspheres prepared with HAP loading up to $50 \mathrm{wt} \%$, most of the larger spheres (above $500 \mu \mathrm{m}$ ) are hollow whereas the smaller microspheres are dense. The formation of hollow spheres is mainly due to the fact that in the case of larger spheres the hydrophylic HAP granules in the dispersed droplet have a tendency to move towards the surface of the droplet, whereas the hydrophobic polymer moves away from the surface of the droplet to the centre part of the droplet. This in turn, makes the centre of the droplet having relatively higher concentration of the polymer. After the removal of DCM from the droplets, each droplet becomes hard with an outer shell containing relatively higher concentration of HAP. During thermal de-binding, the polymer is burnt out from the microspheres and the inner core where the polymer concentration is more becomes a void. But in the case of small spheres, the solidification is faster. The shorter evaporation time restricts the migration of HAP towards the surface thereby giving a uniform distribution of HAP granules in the polymer matrix. The polymer removed during sintering leaves only HAP granules uniformly spread in the spheres which reduces the chances of formation of hollow microspheres.

\section{Conclusions}

Hydroxyapatite microspheres with peculiar spheres-insphere morphology could be prepared using EVA as the binder material. It was observed that by varying the polymer content, the morphology of the sintered hydroxyapatite microspheres could be altered. An initial polymer loading of $30 \mathrm{wt} \%$ was found to be ideal for getting highly spherical microspheres. Of the three different stabilizers used viz. Tween-40, sodium laurate and PVA, only PVA gave regular spherical spheres while others gave irregular shaped agglomerates. The stabilizer concentration did affect the size of the microspheres. As the percentage of PVA increases, the size of the microspheres decreases. The minimum concentration of PVA required for the stabilization of droplets in the aqueous medium was found to be $0 \cdot 1 \mathrm{wt} \%$. Below this concentration, the droplets were not stabilized and finally agglomerated. The increase in the stirring speed causes a decrease in the size of the microspheres. The microspheres produced at $20^{\circ} \mathrm{C}$ gave better spherical geometry compared to that produced at $30^{\circ} \mathrm{C}$. But at an elevated temperature, say, $40^{\circ} \mathrm{C}$, larger irregular shaped agglomerates were produced. The porosity of sintered HAP microspheres decreases with decrease in the binder content. SEM micrographs of HAP/EVA microsphere show that each sphere is, in turn, composed of hydroxyapatite granules of $2-15 \mu \mathrm{m}$ embedded in EVA matrix. It is hoped that this class of HAP microstructured microspheres could be of potential use in bone filling, bone regeneration, drug delivery etc.

\section{References}

Bonfield W, Grynpas M D, Tully A E, Bowman J and Abram J 1981 Biomaterials 2185

Hrdina K E, Halloran J W, Oliveira A and Kaviany M 1998 J. Mater. Sci. 332795

Hsu F Y, Chueh S C and Wang Y J 1999 Biomaterials 201931

Jarcho M, Bolen C H, Thomas M B, Bobich J, Kay J F and Doremus J 1976 J. Mater. Sci. 112027

Jellinek H H G 1955 in Degradation of vinyl polymers (NY: Academic Press, Inc.) Ch. 2

Klein C A P T, Patka P and den Hollander W 1989 Biomaterials 1059 
Kumazawa Y, Yokogawa T, Tsurui H, Miura K and Watanabe K 1992 Nucleic Acids Research 202223

Kunieda K et al 1993 Br. J. Cancer 67668

Matsumoto T, Okazaki M, Inoue M, Yamaguchi S, Kusunose T, Toyonaga T, Hamada Y and Takahashi J 2004 Biomaterials 233807

Paul W and Sharma C P 1999 J. Mater. Sci. Mater. Med. 10 383

Shigeru K, Oku T and Takagi S 1989 J. Ceram. Soc. Jap. Int. Ed. 9796

Sivakumar M and Rao P K 2002 Biomaterials 233175
Sunny M C, Varma H K and Ramesh P 2002 J. Mater. Sci. Mater. Med. 131

Trunec M and Cihlar J 2002 J. Eur. Ceram. Soc. 222231

Varma H K and Sivakumar R 1996 Phosphorus Research Bulletin 635

Velayudhan S, Ramesh P, Sunny M C and Varma H K 2000 Mater. Letts 46142

Wang Y J, Lin F H, Sun J S, Huang Y C, Chueh S C and Hsu F Y 2003 Artif. Org. 27162

Wu T J, Huang H H, Lan C W, Lin H C, Hsu F Y and Wang Y J 2004 Biomaterials 25651 